\title{
Enterococcus faecalis FK-23 affects alveolar-capillary permeability to attenuate leukocyte influx in lung after influenza virus infection
}

\author{
Kazutake Fukada ${ }^{1,2^{*}}$, Daisuke Fujikura ${ }^{1,4}$, Yosuke Nakayama1,3, Masatoshi Kondoh², Takashi Shimada ${ }^{2}$ \\ and Tadaaki Miyazaki ${ }^{1,3}$
}

\begin{abstract}
Infection with influenza A virus, one of the most common life-threatening viruses, causes the accumulation of inflammatory cells in the lung, which is directly correlated with influenza-associated morbidity and mortality. In this study, we investigated the potential of lysozyme-treated Enterococcus faecalis FK-23 (LFK) to prevent influenza in influenza virus-infected mice. C57BL/6N mice were orally administered LFK and intranasally infected with influenza virus A/Puerto Rico/8/34 (H1N1) at lethal doses. After infection with influenza A virus, the survival rate of the LFK-administered mice was significantly higher than that of saline-administered mice. Staining of lung sections with hematoxylin-eosin, and cell counts of lung and bronchoalveolar lavage fluid showed that oral administration of LFK suppressed the excessive infiltration of leukocytes into the lung after viral infection. Extravasation assay revealed that the arrest was mediated by modulation of pulmonary alveolar-capillary permeability. Expression levels of genes involved in matrix degradation, which are correlated with vascular permeability, were downregulated in LFK-administered mice. These findings suggest that stabilizing the integrity of the alveolar-capillary barrier by the administration of LFK improves survival rate.
\end{abstract}

Keywords: H1N1 influenza virus infection, Lactic acid bacteria, Enterococcus faecalis, Alveolar-capillary permeability, Leukocyte influx

\section{Background}

Influenza is a highly contagious acute respiratory disease caused by influenza viruses, which belong to the Orthomyxoviridae family. According to reports issued by the World Health Organization, approximately 5$15 \%$ of the world's population is annually infected with influenza A virus, and 250, 000-500, 000 of these infected patients die each year. Annual immunization is the primary means to protect from influenza virus infection, but this vaccination strategy can be limited by the production time (Boltz et al. 2010). In addition to the vaccination strategy, antiviral therapy is useful to control the spread of influenza. Two classes of antiviral drugs (M2-ion

\footnotetext{
*Correspondence: lab@nichinichi-phar.co.jp

'Division of Bioresources, Research Center for Zoonosis Control, Hokkaido

University, Sapporo 001-0020, Japan

${ }^{2}$ Central Research Laboratories, Nichinichi Pharmaceutical Corporation Ltd., 239-1 Tominaga, Iga, Mie 518-1417, Japan

Full list of author information is available at the end of the article
}

channel inhibitors and neuraminidase inhibitors) have been approved for the prevention and treatment of influenza (Boltz et al. 2010; van der Vries et al. 2011). However, the effectiveness of these antiviral agents may be limited by the rapid emergence of drug-resistant viruses (van der Vries et al. 2011).

Severe influenza virus infection can lead to diffuse alveolar damage, which is characterized by pulmonary edema and the accumulation of inflammatory cells in the lung, with histopathologic features of acute lung injury (ALI) and acute respiratory distress syndrome (ARDS), the most severe form of ALI. These lung injuries directly correlate with influenza-associated morbidity and mortality because of the impairment of gas exchange and respiratory functions. ARDS is characterized by an increase in the permeability of the alveolar-capillary barrier, which is formed by the microvascular endothelium and the alveolar epithelium, leading to an influx of fluid and leukocytes into the alveolar airspace across both the endothelium and the 
epithelium (Nunes 2005). ALI and ARDS were leading causes of death following infections with pandemic 2009 H1N1 and highly pathogenic avian H5N1 influenza viruses (Perrone et al. 2008; Zhang et al. 2012b).

Several experimental studies have shown that influenzainduced death is suppressed by anti-inflammatory agents that counteract the inflammatory response of the hosts without affecting virus replication itself (Darwish et al. 2011; Garcia et al. 2010). These reports indicate that suppression of the heightened inflammatory response to viral infection is important in order to avoid influenza-caused death.

Oral or intranasal administrations of lactic acid bacteria are effective against influenza A virus infection (Izumo et al. 2010; Maeda et al. 2009). These bacterial administrations help to enhance host's immune response that causes the reduction of the viral replication efficiency and/or upregulation of cytokine expression. Previously, we reported that the water-soluble fraction of lysozymetreated lactic acid bacterium Enterococcus faecalis FK-23 (LFK) reduces the mortality associated with influenza A virus infections (Kondoh et al. 2012). However, the mechanism underlying the anti-influenza effect of LFK remains unclear. We previously reported that the oral administration of LFK attenuates the eosinophil influx into the upper airway in a murine allergic model (Zhu et al. 2012) and the inflammatory cell influx into bronchoalveolar lavage fluid (BALF) in a murine asthmatic model (Zhang et al. 2012a). These results implied the possibility of anti-inflammatory effect of LFK during influenza virus infection. In this study, we demonstrate that the administration of LFK reduces mortality after H1N1 viral infection and suppresses the excessive influx of leukocytes, which cause inflammatory reactions, into lungs via modulation of the alveolarcapillary permeability.

\section{Results \\ Reduction of the mortality of influenza virus-infected mice by LFK administration}

To test for preventive effect against influenza, we orally administered LFK to mice at a dose of $15 \mathrm{mg}$ per mouse, once daily, for 6 days before and 17 days after the viral infection, and monitored the survival rate for 17 days after the infection (Figure 1A). In this experiment, the LFK dose was fixed at $15 \mathrm{mg}$ per mouse as previous report in which water-soluble fraction of LFK suspension (15 mg per mouse) was administered to mice (Kondoh et al. 2012). However, in this experiment, LFK suspension rather than water-soluble fraction of LFK was administered for comprehensive understanding of the mechanism for prevention of influenza by LFK. As shown in Figure 1B, $16 \%$ of mice in the control group, which were administered saline orally, survived for 17 days post-infection. In contrast, $45 \%$ of mice administered LFK orally survived after infection. This result indicates that oral administration of LFK provides effective protection from lethal infection by influenza A virus.

Next, we examined whether the improved survival was due to a reduction in the viral replication efficiency in the lung, because oral or intranasal administration of lactic acid bacteria was shown to suppress viral replication efficiency in the lung (Maeda et al. 2009; Izumo et al. 2010). In contrast to these reports, the viral titer in lungs showed no significant difference between the saline- and LFKadministered groups at 3 days, 5 days, and 7 days postinfection (DPI) (Figure 1C). This result suggests that the mechanism by which oral administration of LFK protects against virus-induced death is different from that reported for other lactic acid bacteria.

\section{Suppression of the infiltration of leukocytes into lung after virus-infection by LFK treatment}

Leukocytes, including inflammatory cells, mononuclear cells, and lymphocytes, infiltrate into the lung area after viral infection (Kohlmeier and Woodland 2009; Fukushi et al. 2011). To gain insight into the protective mechanism underlying LFK activity, we stained the lung sections using hematoxylin-eosin (HE). This staining revealed that infiltration of leukocytes into the pulmonary parenchyma was suppressed at DPI-7 in the LFK-administered group, whereas infiltration of leukocytes in the pulmonary parenchyma and alveolar collapse were observed in the saline-administered group (Figure 2A and Additional file 1: Figure S1). To confirm that LFK suppresses the infiltration of leukocytes, we homogenized the removed lungs and counted the number of whole lung cells by using a microscope. In the saline-administered group, the number of lung cells increased after viral infection and reached a maximum at DPI-7 (Figure 2B). Unlike in the control group, the number of these cells in the LFK-administered group was significantly suppressed at DPI-5, DPI-7, and DPI-10 (Figure 2B). Similar results were obtained for BALF cells. The total cell number in the BALF was suppressed in the LFK-administered group at DPI-5 compared to that in the saline-administered group (Figure 2D).

\section{Upregulation of type II pneumocyte number by LFK administration}

Surprisingly, the number of whole lung cells at DPI-0 was significantly higher in mice that were administered with LFK for 6 days before viral infection than in the saline-administered group (Figure 2B). The alveolar septa consists of 2 types of pneumocytes (type I and II), connective tissues, and blood vessels (Rogers 2010). We performed an immunohistochemical analysis because, on the basis of our observation of the HE-stained lung sections at DPI-0 (Additional file 2: Figure S2A), we expected an increase in the number of type II pneumocytes. Staining with type II 
A

LFK (15 mg/head/day)

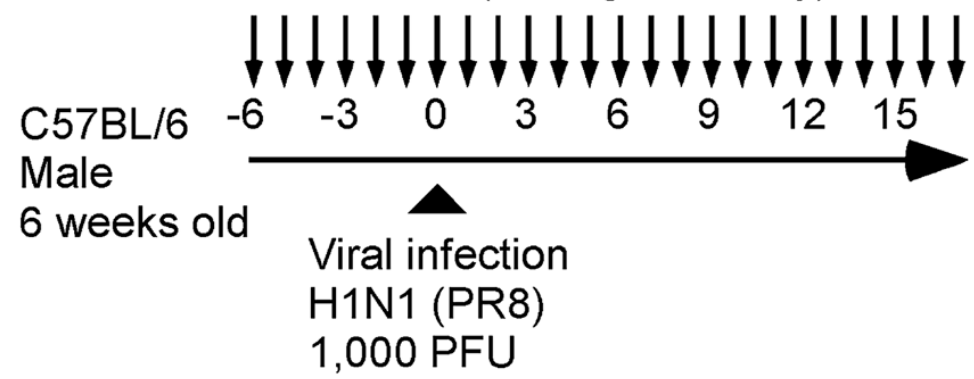

B
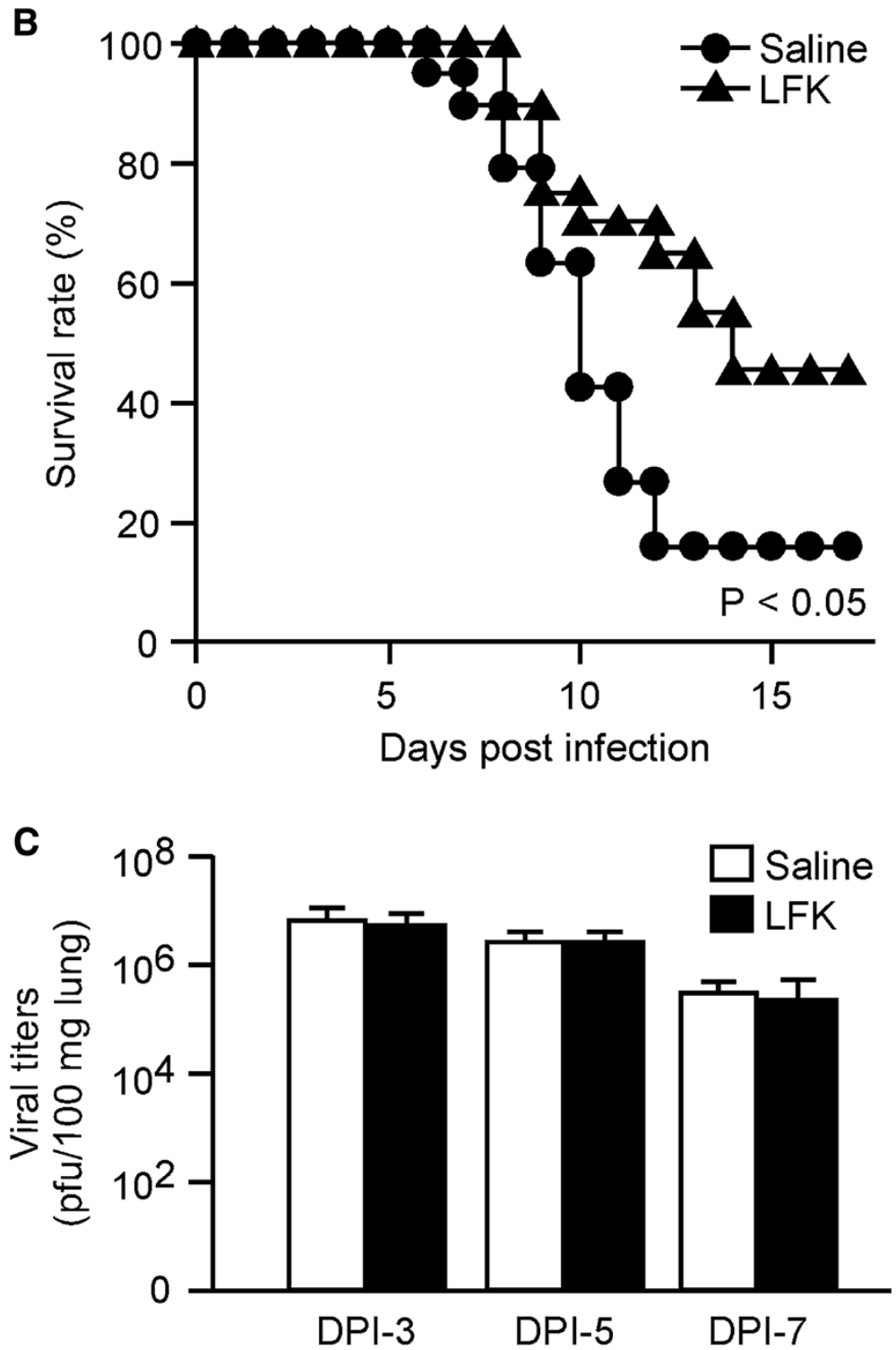

Figure 1 (See legend on next page.) 
(See figure on previous page.)

Figure 1 Administration of LFK protects mice from influenza virus PR8-induced death. (A) The experimental protocol for oral administration and infection with influenza A/PR/8. The vertical arrows indicate oral administration of LFK (15 mg/head, dissolved in $200 \mu \mathrm{l} \mathrm{saline)} \mathrm{or} \mathrm{saline} \mathrm{from} \mathrm{day} \mathrm{-6} \mathrm{to}$ day 17. The triangle on day 0 indicates the inoculation with PR8 (1,000 PFU/mouse). (B) Survival rate of mice after the infection with PR8 (salineadministered group: circle, $n=19$; LFK-administered group: triangle, $n=20$ ). Statistically significant difference between saline and control mice is indicated by $P$ value $(P<0.05$ by the log-rank test). (C) Viral titers in the lungs after inoculation with PR8. Mean viral titers \pm standard deviations are shown. Each group consists of 6 to 7 mice.

pneumocyte marker (proSP-C, prosurfactant protein-C) showed that the number of type II pneumocyte was upregulated in the LFK-administered group compared to that in the saline-administered group (Additional file 2: Figures S2B and S2C).

\section{Suppression of cellular infiltration independent of cell type} Next, we evaluated which types of cells infiltrate into the lung area after viral infection. Cell populations of each leukocyte in the lung were analyzed at DPI-0, DPI-3, DPI-5, DPI-7, and DPI-10 using a flow cytometer. In the early phase of infection with influenza virus, innate immune cells, such as monocytes, macrophages, and neutrophils, were recruited to the lung at DPI-5 and DPI-7 (Figure $2 \mathrm{C}$ ). Thereafter, $\mathrm{T}$ cell migration was observed (Figure 2C). However, there was no significant difference between the saline- and LFK-administered groups in the population of the infiltrating cells. A similar result was obtained for the cell population in the BALF at DPI-5. The saline- and LFK-administered groups showed no significant difference in the population of leukocytes that infiltrated the alveolar space (Figure 2E). These results indicate that the suppression of cellular infiltration into the lung occurred in all types of leukocytes that we analyzed.
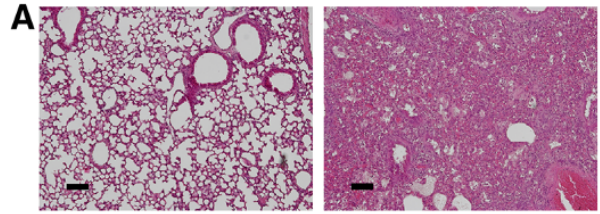

B

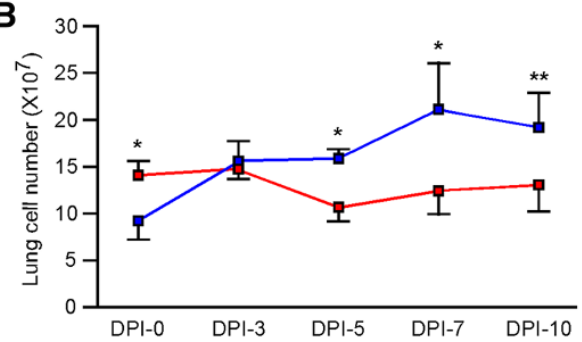

D
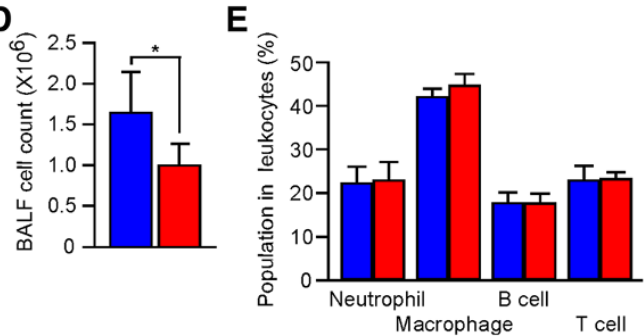

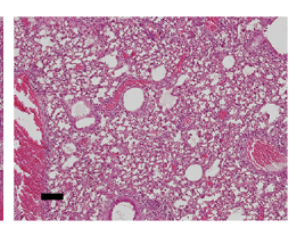

C

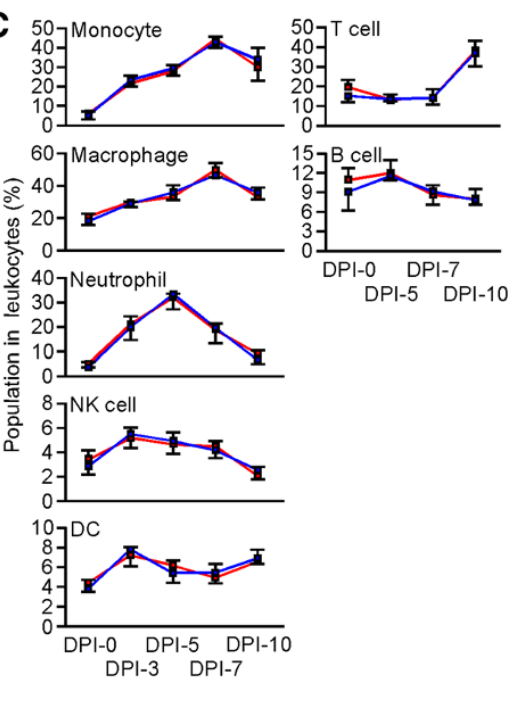

Figure 2 Administration of LFK suppresses the leukocytes infiltration into the lungs after PR8 viral infection. (A) Histology of lung tissue section stained with HE at DPI-7 (left: non-infected control, middle: saline-administered mice at DPI-7, right: LFK-administered mice at DPI-7). Original magnification is X10. Scale bars indicate $100 \mu \mathrm{m}$. (B) The lung cells were isolated at DPI-0, DPI-3, DPI-5, DPI-7 and DPI-10, and the absolute number of lung cells was counted (*: $P<0.01,{ }^{* *}: P<0.05$, Student's $t$ test). Blue and red squares indicate saline- and LFK-administered groups, respectively (DPI-0: saline $n=7$, LFK $n=7$; DPI-3: saline $n=5$, LFK $n=4$; DPI-5: saline $n=5$, LFK $n=4$; DPI-7: saline $n=5$, LFK $n=5$; DPI-10: saline $n=3$, LFK $n=5$ ). (C) The change in lung cell population (blue: saline-administered group, red: LFK-administered group) was analyzed through a flow cytometer (DPI-0: saline $n=7$, LFK $n=7$; DPI-3: saline $n=5$, LFK $n=4$; DPI-5: saline $n=5$, LFK $n=4$; DPI-7: saline $n=5$, LFK $n=5$; DPI-10: saline $n=3$, LFK $n=5$ ). (D) The total number of BALF cells at DPI-5 was counted (blue: saline-administered group, $n=8$; red: LFK-administered group, $n=6$; ${ }^{*}, \mathrm{P}<0.05$, Student's $t$ test). (E) Cell population in BALF was analyzed through a flow cytometer (blue: saline-administered group, $n=8$; red: LFK-administered group, $n=6$ ). 


\section{Gene expression of chemokines and cytokines after virus infection}

Chemokines regulate the trafficking of various types of leukocytes, and cytokines can induce the production of chemokines (Kohlmeier and Woodland 2009). Therefore, we next evaluated the mRNA expression level of cytokines and chemokines in the lung during the course of viral infection. The expression level of various Th1 cytokines, pro-inflammatory cytokines, CCL-chemokine ligands, and CXCL-chemokine ligands were elevated after the viral infection (Figure 3A, 3B, and Additional file 3: Figure S3A and $\mathrm{S} 3 \mathrm{~B})$. However, no significant difference in the expression level of cytokines and chemokines was observed except for CXCL4 (platelet factor 4), which on its own does not show any chemotactic activity to leukocytes (Kasper and Petersen 2011).

\section{Suppression of virus-induced alveolar-capillary permeability by LFK treatment}

Next, we examined whether the suppression of leukocyte migration was due to the changes in pulmonary alveolarcapillary permeability, because pulmonary inflammation during viral infection is closely correlated with the breakdown of pulmonary barrier integrity (Steinberg et al. 2011; Fukushi et al. 2011). The change in alveolar-capillary permeability was assessed by monitoring the extravasation of Evans blue dye into the BALF. As shown in Figure 4A, increases in virus-induced Evans blue accumulation were suppressed at DPI-7 in the LFK-administered mice. This indicates that the administration of LFK suppresses viral infection-induced pulmonary permeability, and that the suppression of inflammatory cell influx by LFK is due to stabilization of the alveolar-capillary barrier integrity.

\section{Modulation of gene expression controlling the vascular permeability by LFK administration}

Mechanisms that control vascular permeability during inflammation have been reported. Matrix metalloproteinases (MMPs) are important regulatory enzymes in pro-inflammatory pathways, and their expression and activities are typically increased during the inflammatory process. MMPs degrade major components of the vascular basement membrane and extracellular matrix, such as gelatin and collagen, in the alveolar-capillary barrier (Manicone and McGuire 2008). Therefore, we investigated the mRNA expression level of MMPs in the lung during the course of viral infection. MMP-7 expression following viral infection was suppressed in the LFKadministered group at DPI-7 (Figure 4B). For the other MMPs, a decreasing trend was observed in the LFKtreated group at DPI-7 (Figure 4B). These results suggest that the downregulation of MMP expression by LFKadministration could attenuate the vascular permeability caused by viral infection.

\section{Discussions}

A number of probiotic bacteria, including Bifidobacteria and lactic acid bacteria such as Lactobacillus, have an anti-influenza effect that is achieved by the reduction of viral replication efficiency and/or upregulation of Th1 cytokine expression through the activation of the host's immune system (Harata et al. 2010; Yasui et al. 2004; Maeda et al. 2009; Izumo et al. 2010). We previously reported that oral administration of the soluble components of LFK, lysozyme-treated lactic acid bacteria E. faecalis FK-23, has an anti-influenza effect by upregulation of the anti-inflammatory cytokine IL-10 in lung (Kondoh et al. 2012). However, administration of the LFK suspension did not markedly upregulate IL-10 expression in this study (Figure 3A). This discrepancy could be due to the differences in composition between administered samples. In this report, LFK suspension was administered to mice, while the supernatant of LFK suspension after centrifuge was used in the previous report (Kondoh et al. 2012). The present study demonstrated that LFK suspension has the ability to neither repress the viral replication, nor enhance the immune system in the lungs (Figures $1 \mathrm{C}$, 3, and Additional file 3: Figure S3). These results indicate that the mechanism by which oral administration of LFK protects against virus-induced death is different from that reported for other probiotic bacteria.

Pneumocytes play pivotal roles in pulmonary function: (1) lung surfactants secreted by pneumocytes prevent alveolar collapse (Griese 1999), (2) type II pneumocytes are progenitor cells for alveolar epithelium during injuries (Mason 2006), and (3) type I pneumocytes are responsible for oxygen and carbon dioxide exchange (Mason 2006). In Figure S2, we demonstrated that oral administration of LFK increases the number of type II pneumocytes, which act as alveolar epithelial stem cells (Fehrenbach 2001). However, further experiments, such as immunohistochemical analysis using antibodies against proliferation marker and mitogen, are required to clear how the proliferation is induced by LFK. Influenza virus infection against type II pneumocyte causes damage to the alveolar epithelial cells and/or the cell death (Matute-Bello et al. 2001; van Riel et al. 2007; Loosli et al. 1975). The damage and/or loss result in disturbances in fluidity at the alveolar-capillary border, which is associated with gas exchange inefficiency and pulmonary vascular permeability (Mura et al. 2010; Ware and Matthay 2000; Matute-Bello et al. 2001). Therefore, the increase in the number of type II pneumocytes in the LFK-administered group suggests that LFK might have the effect of improving respiratory function and epithelial barrier function by the proliferation and/or differentiation of type II pneumocyte during viral infection. Another possibility is that the increase in the number of type II pneumocytes might cause the lower multiplicity of the virus in the virus-infected pneumocyte, which leads to the 

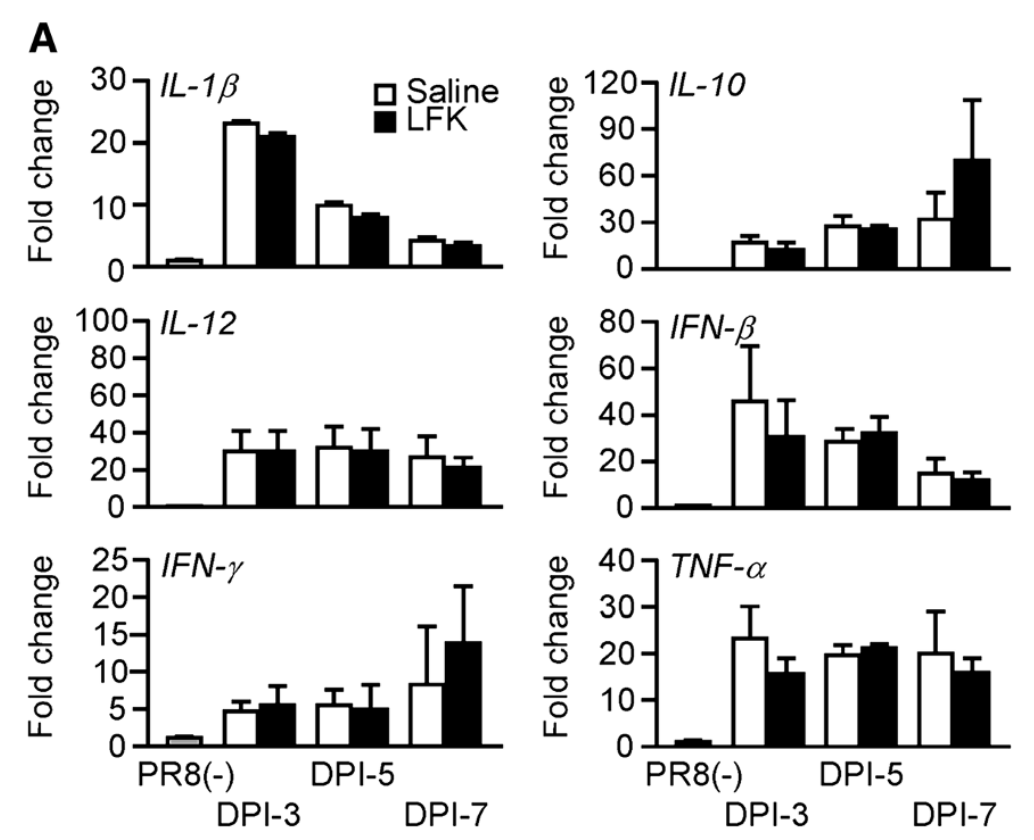

B
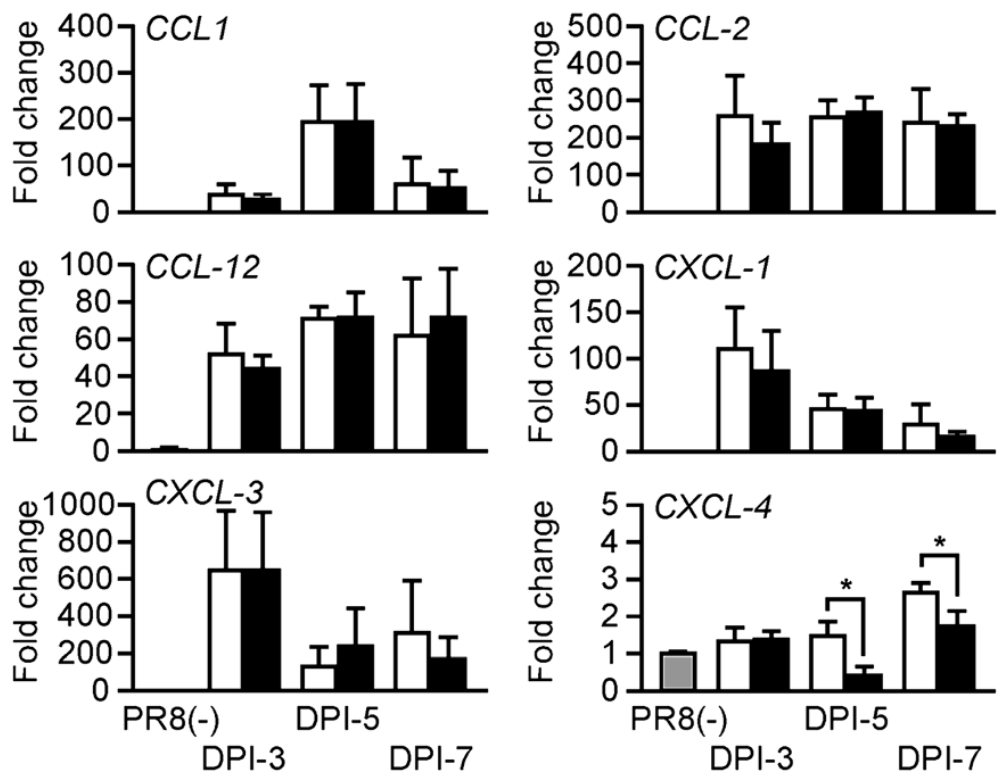

Figure 3 Cytokine and chemokine mRNA expression levels during the course of the viral infection. (A) Cytokine mRNA expression level in lung. (B) Chemokine mRNA expression level in lung. Each value is expressed as fold change compared to non-infected control mice $(*$ : $P<0.01$, Student's $t$ test). Gray, white, and black columns indicate non-infected control $(n=3)$, saline-administered $(n=6)$, and LFK-administered ( $n=6)$ groups, respectively.

repression of cell death of type II pneumocyte and successive vascular permeability. However, further analysis of the induction of cell death, proliferation, and differentiation in the type II pneumocyte is required to unmask the role of the increased pneumocyte in LFK-administered mice during the influenza virus infection.

Cytokines and successive chemokines are produced following influenza virus infection in the lungs as early anti-viral responses (Kohlmeier and Woodland 2009). In the present study, the expression of cytokines and chemokines after viral infection were not modulated by the treatment with LFK, except for CXCL4 (Figure 3), although the reported probiotic bacteria regulate the expressions as a viral defense mechanism (Maeda et al. 2009; Yasui et al. 2004). This is supported by our unpublished data that show that elimination of peptidoglycan 

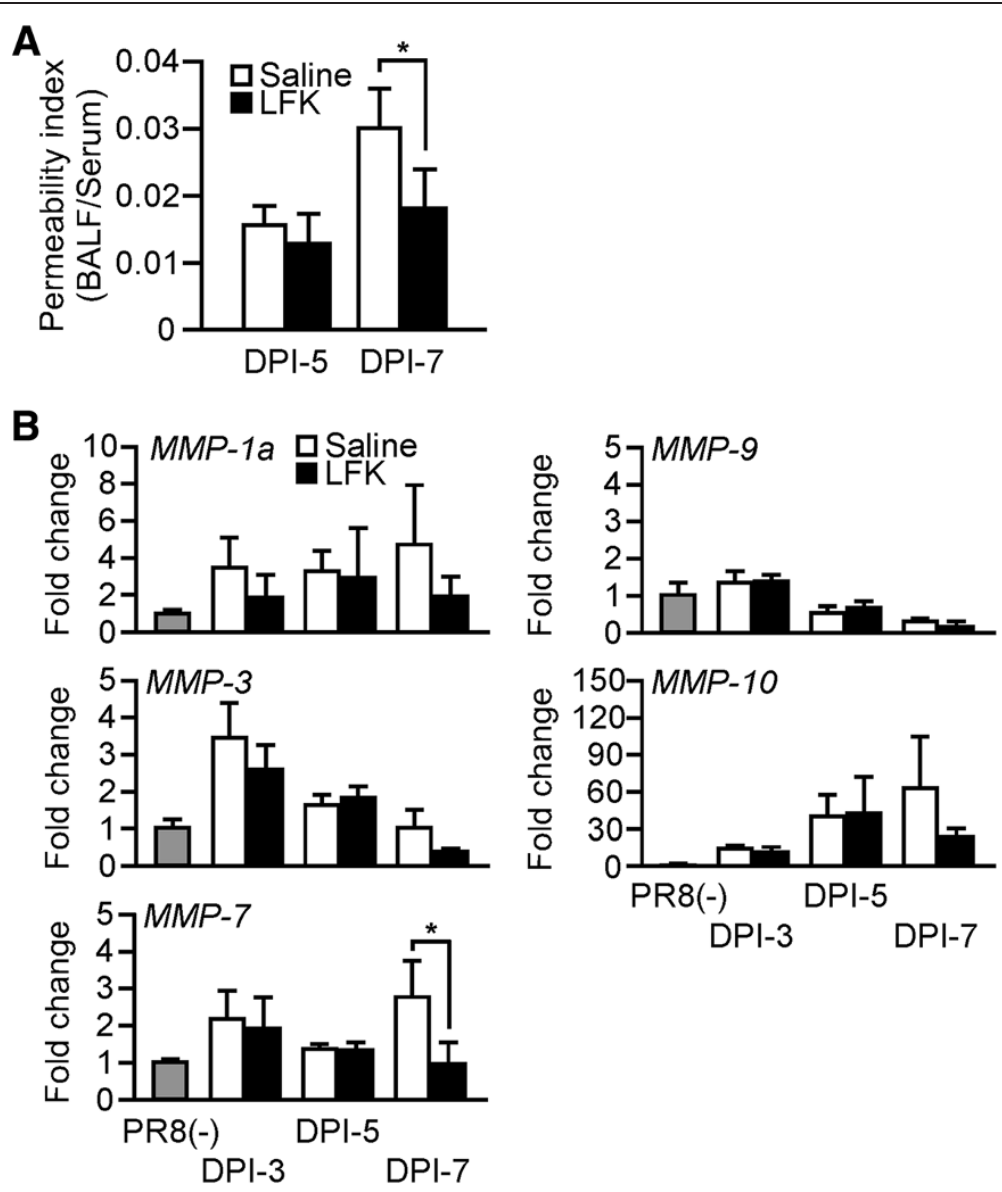

Figure 4 Administration of LFK suppresses the virus-induced alveolar-capillary permeability in lungs. (A) Alveolar-capillary permeability was assessed by extravasation of Evans blue dye into BALF at DPI-5 and DPI-7. Permeability index was determined from the dye content of BALF compared with serum (DPI-5: saline $n=5$, LFK $n=6$; DPI-7: saline $n=5$, LFK $n=5$; : P $<0.05$, Student's $t$ test). (B) MMP mRNA expression level in lung. Each value is expressed as fold change compared to non-infected control mice (*: $P<0.05$, Student's $t$ test). Gray, white, and black columns indicate non-infected control $(n=4)$, saline-administered $(n=4)$, and LFK-administered $(n=4)$ groups, respectively.

from the cell of $E$. faecalis FK-23 by enzymatic degradation results in loss of the ability to produce various cytokines in ex vivo culture of the splenocytes from mice (data not shown). Increased influx of leukocytes into alveolar space occurs after viral infection, and leukocyte extravasation from the blood to the inflamed tissue is a controlled process involving rolling, adhesion, and migration (Weber et al. 2007). While CXCL4 shows no chemotactic activity against leukocytes, CXCL4 enhances monocyte adhesion to the endothelium and neutrophil extravasation to the inflamed lung via heterophilic interaction with CCL5 (Grommes et al. 2012; von Hundelshausen et al. 2005; Kasper and Petersen 2011). Therefore, our results suggest that the suppression of leukocyte migration into the lung by the administration of LFK is partially mediated by the downregulation of CXCL4 expression.

MMPs induced by influenza virus infection degrade the components of the vascular basement membrane and extracellular matrices, and the resulting dysfunction of the alveolar-capillary barrier contributes to pathogenesis (Ng et al. 2012; Wang et al. 2010; Davey et al. 2011; Manicone and McGuire 2008). MMP-7 is reported to degrade not only VE-cadherin but also E-cadherin, which are major components of endothelial and epithelial adherens junctions, respectively (McGuire et al. 2003; Ichikawa et al. 2006). These adherens junctions act as gatekeepers for the passage of leukocytes during inflammation. Furthermore, stabilization of vascular barrier integrity by upregulating the expression of the VE-cadherin mRNA reduces mortality after H5N1 viral infection and suppresses leukocyte influx into the lung (London et al. 2010). Therefore, our results suggest that downregulation of the expressions of MMP-7 and other MMPs by LFK attenuates not only the breakdown of adherens junctions of alveolar epithelial and vascular endothelial cells, but also degradation of the vascular basement membrane and extracellular matrices. However, further analyses for protein level of MMP and MMP-mediated degradation of target proteins would be 
required for comprehensive understanding of the effect of LFK for the prevention of influenza.

The components of LFK to exert the anti-influenza effect remain unidentified. Since a recent study has demonstrated that the soluble peptidoglycan of commensal bacteria translocates from the intestine to the systemic circulation (Clarke et al. 2010), one possibility is that the lysozyme-degraded components of peptidoglycan could translocate to the lungs, thereby modulating the alveolarcapillary barrier. Identification of the active components in LFK will be essential to further understand the precise anti-influenza mechanism.

In this study, we demonstrated that the administration of LFK improved survival rates and suppressed the infiltration of leukocytes into the lung after viral infection. Several factors could contribute to the effect of LFK against influenza virus infection. These include the suppression of leukocyte infiltration into the lungs by both the downregulation of CXCL4 expression and the modulation of pulmonary endothelial-epithelial permeability via the increase of type II pneumocytes and the repression of MMPs expression. Our findings support the notion that suppression of the breakdown of the alveolar-capillary barrier and the subsequent leukocyte influx into the lung would improve the survival rate after viral infection. Stabilization of pulmonary alveolar-capillary barrier integrity using bacterial components might be a useful strategy for managing seasonal and pandemic influenza.

\section{Methods}

\section{LFK preparation}

E. faecalis strain FK-23 was cultured in broth medium containing $2.5 \%$ of glucose, $1.4 \%$ of yeast extract, $0.8 \%$ of peptone, and $4.4 \%$ of $\mathrm{K}_{2} \mathrm{HPO}_{4}$ for $18 \mathrm{~h}$ at $37^{\circ} \mathrm{C}$, and the cultures were harvested by centrifugation. After washing with distilled water, the bacteria were treated with lysozyme, and then the reaction mixture was heated to $110^{\circ} \mathrm{C}$ for $10 \mathrm{~min}$ before lyophilization as described previously (Kondoh et al. 2012). LFK (15 mg/head, dissolved in $200 \mu \mathrm{l}$ saline) or saline $(200 \mu \mathrm{l})$ was orally administered using a feeding needle once a day.

\section{Virus preparation}

An influenza virus strain, A/Puerto Rico/8/34 (H1N1; PR8) was used in this study. The infectious materials were handled in a biosafety level 2 facility under approved protocols in accordance with guidelines of Hokkaido University. The virus was prepared as described previously (Kondoh et al. 2012). In brief, the virus was propagated in the allantoic cavities of 10-day-old embryonated chicken eggs at $35^{\circ} \mathrm{C}$ for $48 \mathrm{~h}$, and then was concentrated and purified by density gradient centrifugation. The purified virus was suspended in phosphate-buffered saline (PBS) and stored at $-80^{\circ} \mathrm{C}$ until use.

\section{Mice and viral infection}

Male C57BL/6N mice (6 weeks old) were purchased from CLEA Japan. Mice were housed in isolator cages in a biosafety level 2 room ( $12 \mathrm{~h}$ light/dark cycle) with free access to standard diet (CE-2; CLEA Japan) and tap water. We performed animal care and experiments in accordance with guidelines and approval of the Animal Care and Use Committee of Hokkaido University (080231). All surgery was performed under isoflurane anaesthesia. Mice were lightly anesthetized with isoflurane (Dainippon Pharmaceutical, Osaka, Japan) and inoculated intranasally with $10^{3} \mathrm{PFU}$ at $50 \mu \mathrm{l}$ in both nostrils on day 0 . The survival rate and body weight were monitored daily until 17 days after the viral infection. To minimize suffering after viral challenge, mice were carefully observed each day and mice reaching approved endpoint criteria were euthanized by overdose of isoflurane. Mice were euthanized by overdose of isoflurane on day 17 after the infection.

\section{Viral titration}

Plaque forming assay was performed as described previously (Fukushi et al. 2011) with some modifications of the protocol. Mice were sacrificed by isoflurane inhalation and lung was removed from the mice. The lungs were completely homogenized in 1X MEM medium using Micro smash (Tomy Seiko, Tokyo, Japan), and the homogenates were serially diluted with cold PBS. For a plaque assay, Madin-darby canine kidney (MDCK) cells were plated in a flat bottomed 12 well plate $24 \mathrm{~h}$ before infection. Supernatants from lung homogenates serially diluted were used to infect the confluent MDCK cells at $37^{\circ} \mathrm{C}$ for $1 \mathrm{~h}$. The cells were washed and subsequently overlaid with MEM mixed with $0.8 \%$ Bacto-agar (Difco, Sparks, USA) in the presence of trypsin $(5 \mu \mathrm{g} / \mathrm{ml})$. The plates were incubated at $35^{\circ} \mathrm{C}$ for 2 days and the plaques were counted.

\section{Histological analysis}

Mice were sacrificed by isoflurane inhalation and lung was removed from the mice. Removed lungs were immediately fixed in 10\% PBS-buffered formalin. Paraffinembedded tissues were sectioned to a thickness of $4 \mu \mathrm{m}$ and stained with hematoxyline and eosin (Merck, USA) using standard histological techniques. For immunehistochemical study, the paraffin-embedded tissue sections were incubated with anti-prosurfactant protein $\mathrm{C}$ antibody which is a type II pneumocyte marker (Millipore, Billerica, USA), incubated with horseradish peroxidase labeled polymer anti rabbit system (DAKO, Glostrup, Denmark), developed with envision + system HRP labeled polymer anti-rabbit (DAKO Glostrup, Denmark), and counterstained with hematoxylin (Merck, USA) in accordance to manufacturer's instructions. The proportion of prosurfactant protein $\mathrm{C}$ positive cells was calculated by 
counting the number of hematoxyline stained cells and its positive cells in 6 random microscopic fields at a magnification of 40 .

\section{Flow cytometric analysis of lung and BALF cells}

Mice were sacrificed by isoflurane inhalation and lung was removed from the mice. Lung tissue was homogenized using gentleMACS dissociator (Miltenyi Biotech, Bergisch Gladbach, Germany) in C tube (Miltenyi Biotech, Bergisch Gladbach, Germany) containing HEPES buffer (pH 7.4) with $2 \mu \mathrm{g} / \mathrm{ml}$ of collagenase-D (Roche, Basel, Switzerland) and $40 \mathrm{U} / \mathrm{ml}$ of DNase I (Takara bio, Otsu, Japan), and then incubated for $30 \mathrm{~min}$ at $37^{\circ} \mathrm{C}$ with gentle rotation. After centrifugation, cells were treated with lysis buffer (BD Biosciences, Franklin lakes, USA) according to manufacture's instruction. The bronchoalveolar lavage fluid (BALF) cells were collected using a 24G catheter (Terumo, Tokyo, Japan) by insertion of PBS from the trachea. Number of lung and BALF cells was counted using a Neubauer hemocytometer (Erma, Tokyo, Japan). Cells were incubated with monoclonal antibody 2.4G2, and stained with FITCconjugated anti-CD3e (BD Biosciences, clone 145-2C11) for T-cell $\left(\mathrm{CD}^{+} \mathrm{e}^{+}\right)$analysis, with FITC-CD11b (BD Biosciences, clone MI/70) and PE-Ly6G (BD Biosciences, clone 1A8) for neutrophils (CD11b ${ }^{\text {high }}$ Ly6G ${ }^{\text {high }}$ ) and monocytes $\left(\mathrm{CD} 11 \mathrm{~b}^{\text {high }}\right.$ Ly6G $\left.{ }^{\text {med}}\right)$ analyses, with FITC-CD49b (BD Biosciences, clone DX5) for NK cells $\left(\mathrm{CD}_{4} 9 \mathrm{~b}^{+}\right)$analysis, with FITC-B220 (Biolegend, clone RA3-6B2) for B cells $\left(B 220^{+}\right)$ analysis, or with APC-CD11c (Biolegend, clone N418) and $\mathrm{PE} / \mathrm{Cy} 7-\mathrm{F} 4 / 80$ (Biolegend, clone BM8) for dendritic cell $\left(\mathrm{CD} 11 \mathrm{c}^{+} \mathrm{F} 4 / 80^{-}\right)$and macrophages $\left(\mathrm{F} 4 / 80^{+}\right)$analyses. The stained cells were analyzed with FACSCanto II flow cytometer (BD Biosciences).

\section{Real time PCR}

Real time PCR reaction was performed as described previously (Kondoh et al. 2012). In brief, total RNA was extracted from whole lung homogenates using TRIzol reagent (Invitrogen, Carlsbad, USA) and Micro smash (Tomy Seiko, Tokyo, Japan), and then treated with DNase I (Takara bio, Otsu, Japan). The cDNA was synthesized using oligo dT20 primers (Toyobo, Osaka, Japan), random primers (Toyobo, Osaka, Japan) and ReverTra Ace (Toyobo, Osaka, Japan). Each procedure was performed according to the manufacture's instructions. Real time PCR was performed using SYBR Premix Ex Taq II (Takara bio, Otsu, Japan) with the MX3000P real time PCR system (Stratagene, La Jolla, USA). Cycling conditions were used as: $95^{\circ} \mathrm{C}$ for $10 \mathrm{sec}$ to activate DNA polymerase, followed by 40 cycles of $95^{\circ} \mathrm{C}$ for $5 \mathrm{sec}$ and $60^{\circ} \mathrm{C}$ for $30 \mathrm{sec}$. Expression levels of each mRNA were represented as relative expression amounts, which were normalized with glyceraldehyde3-phosphate dehydrogenase (GAPDH).

\section{Measurement of alveolar-capillary permeability}

Alveolar-capillary leakage after viral infection was determined using Evans blue dye as described previously (Rhein et al. 2008). Two hours before killing, mice were injected intravenously via retro orbital sinus under isoflurane anaesthesia with $0.2 \mathrm{ml}$ of $5 \mathrm{mg} / \mathrm{ml}$ Evans blue in PBS. BALF and serum were collected after killing mice with isoflurane and the optical density was determined at $600 \mathrm{~nm}$. The permeability changes were evaluated by the BALF/serum concentration ratio.

\section{Statistical analysis}

The data are expressed as means \pm standard deviations (SD). A student's $t$ test was used for statistical analysis. The Kaplan-Meier method with the log-rank test was used for analysis of mortality. P value of $<0.05$ was considered to be significant.

\section{Additional files}

Additional file 1: Figure S1. Histology of lung tissue section stained with HE at DPI-7. Original magnification is X20 (A: non-infected control, B: saline-administered mice at DPI-7, C: LFK-administered mice at DPI-7). Scale bars indicate $100 \mu \mathrm{m}$. In each group, two fields, which were taken from lung section of different mice, are shown.

Additional file 2: Figure S2. Increased number of Type ॥ pneumocyte by the administration of LFK. (A) Histology of lung tissue section stained with HE at DPI-0 (top: saline-administered mice, bottom: LFK-administered mice). Original magnification is X4. Scale bars indicate $250 \mu \mathrm{m}$. (B) Immunohistochemical staining of lung tissue section at DPI-0 using prosurfactant protein C (proSP-C) antibody (top: saline-administered mice, bottom: LFK-administered mice). Arrowheads indicate examples of proSP-C-positive cells. Original magnification is X40. Scale bars indicate $50 \mu \mathrm{m}$. (C) The proportion of proSP-C positive cells was counted in 6 random microscopic fields for each group at a magnification of $X 40$ (blue: saline, red: LFK; ${ }^{*}: P<0.01$, Student's $t$ test).

Additional file 3: Figure S3. Cytokine and chemokine mRNA expression levels during the course of the viral infection. (A) Cytokine mRNA expression level in lung. (B) Chemokine mRNA expression level in lung. Each value is expressed as fold change compared to non-infected control mice $(*$ : $P<0.01$ Student's $t$ test). Black, blue, and red columns indicate non-infected control $(n=3)$, saline-administered $(n=6)$, and LFK-administered $(n=6)$ groups, respectively.

\section{Abbreviations}

ALI: Acute lung injury; ARDS: Acute respiratory distress syndrome;

BALF: Bronchoalveolar lavage fluid; DPI: Days post-infection; HE: Hematoxylineosin; LFK: Lysozyme-treated Enterococcus faecalis FK-23; MDCK: Madin-darby canine kidney; MMP: Matrix metalloproteinase; SP-C: Prosurfactant protein-C.

Competing interests

The authors declare that they have no competing interests.

\section{Authors' contributions}

KF and TM conceived and designed experiments. KF performed the experiments. DF and YN participated in the flow cytometric analysis. YN participated in the tissue section analysis. MK and TS participated in the survival experiment. KF drafted the manuscript. TS and TM revised the manuscript. All authors read and approved the final manuscript.

\section{Acknowledgements}

We thank all colleagues in Hokkaido University Research Center for Zoonosis Control for helpful discussions. This work was supported by Grant of Global 
COE Program, "International Collaboration Centers for Zoonosis Control", Ministry of Education, Science, Sports and Culture.

\section{Author details}

'Division of Bioresources, Research Center for Zoonosis Control, Hokkaido University, Sapporo 001-0020, Japan. ${ }^{2}$ Central Research Laboratories, Nichinichi Pharmaceutical Corporation Ltd., 239-1 Tominaga, Iga, Mie 518-1417, Japan. ${ }^{3}$ Department of Probiotics Immunology, Institute for Genetic Medicine, Hokkaido University, Sapporo 060-0815, Japan. ${ }^{4}$ Division of Infection and Immunity, Research Center for Zoonosis Control, Hokkaido University, Sapporo 001-0020, Japan.

\section{Received: 18 April 2013 Accepted: 13 June 2013}

Published: 20 June 2013

\section{References}

Boltz DA, Aldridge JR Jr, Webster RG, Govorkova EA (2010) Drugs in development for influenza. Drugs 70(11):1349-1362

Clarke TB, Davis KM, Lysenko ES, Zhou AY, Yu Y, Weiser JN (2010) Recognition of peptidoglycan from the microbiota by Nod1 enhances systemic innate immunity. Nat Med 16(2):228-231

Darwish I, Mubareka S, Liles WC (2011) Immunomodulatory therapy for severe influenza. Expert Rev Anti Infect Ther 9(7):807-822

Davey A, McAuley DF, O'Kane CM (2011) Matrix metalloproteinases in acute lung injury: mediators of injury and drivers of repair. Eur Respir J 38(4):959-970

Fehrenbach H (2001) Alveolar epithelial type II cell: defender of the alveolus revisited. Respir Res 2(1):33-46

Fukushi M, Ito T, Oka T, Kitazawa T, Miyoshi-Akiyama T, Kirikae T, Yamashita M, Kudo K (2011) Serial histopathological examination of the lungs of mice infected with influenza A virus PR8 strain. PLoS One 6(6):e21207

Garcia CC, Guabiraba R, Soriani FM, Teixeira MM (2010) The development of antiinflammatory drugs for infectious diseases. Discov Med 10(55):479-488

Griese M (1999) Pulmonary surfactant in health and human lung diseases: state of the art. Eur Respir J 13(6):1455-1476

Grommes J, Alard JE, Drechsler M, Wantha S, Morgelin M, Kuebler WM, Jacobs M, von Hundelshausen P, Markart P, Wygrecka M, Preissner KT, Hackeng TM, Koenen RR, Weber C, Soehnlein O (2012) Disruption of platelet-derived chemokine heteromers prevents neutrophil extravasation in acute lung injury. Am J Respir Crit Care Med 185(6):628-636

Harata G, He F, Hiruta N, Kawase M, Kubota A, Hiramatsu M, Yausi H (2010) Intranasal administration of Lactobacillus rhamnosus GG protects mice from H1N1 influenza virus infection by regulating respiratory immune responses. Lett Appl Microbiol 50(6):597-602

Ichikawa Y, Ishikawa T, Momiyama N, Kamiyama M, Sakurada H, Matsuyama R, Hasegawa S, Chishima T, Hamaguchi Y, Fujii S, Saito S, Kubota K, Hasegawa S, Ike H, Oki S, Shimada H (2006) Matrilysin (MMP-7) degrades VE-cadherin and accelerates accumulation of beta-catenin in the nucleus of human umbilical vein endothelial cells. Oncol Rep 15(2):311-315

Izumo T, Maekawa T, Ida M, Noguchi A, Kitagawa Y, Shibata H, Yasui H, Kiso Y (2010) Effect of intranasal administration of Lactobacillus pentosus S-PT84 on influenza virus infection in mice. Int Immunopharmacol 10(9):1101-1106

Kasper B, Petersen F (2011) Molecular pathways of platelet factor 4/CXCL4 signaling. Eur J Cell Biol 90(6-7):521-526

Kohlmeier JE, Woodland DL (2009) Immunity to respiratory viruses. Annu Rev Immunol 27:61-82

Kondoh M, Fukada K, Fujikura D, Shimada T, Suzuki Y, Iwai A, Miyazaki T (2012) Effect of water-soluble fraction from lysozyme-treated Enterococcus faecalis FK-23 on mortality caused by influenza A virus in mice. Viral Immunol 25(1):86-90

London NR, Zhu W, Bozza FA, Smith MC, Greif DM, Sorensen LK, Chen L, Kaminoh Y, Chan AC, Passi SF, Day CW, Barnard DL, Zimmerman GA, Krasnow MA, Li DY (2010) Targeting Robo4-dependent Slit signaling to survive the cytokine storm in sepsis and influenza. Sci Transl Med 2 (23):23ra19

Loosli CG, Stinson SF, Ryan DP, Hertweck MS, Hardy JD, Serebrin R (1975) The destruction of type 2 pneumocytes by airborne influenza PR8-A virus; its effect on surfactant and lecithin content of the pneumonic lesions of mice. Chest 67(2 Suppl):7S-14S

Maeda N, Nakamura R, Hirose Y, Murosaki S, Yamamoto Y, Kase T, Yoshikai Y (2009) Oral administration of heat-killed Lactobacillus plantarum L-137 enhances protection against influenza virus infection by stimulation of type I interferon production in mice. Int Immunopharmacol 9(9):1122-1125

Manicone AM, McGuire JK (2008) Matrix metalloproteinases as modulators of inflammation. Semin Cell Dev Biol 19(1):34-41

Mason RJ (2006) Biology of alveolar type II cells. Respirology 11(Suppl):S12-S15

Matute-Bello G, Winn RK, Jonas M, Chi EY, Martin TR, Liles WC (2001) Fas (CD95) induces alveolar epithelial cell apoptosis in vivo: implications for acute pulmonary inflammation. Am J Pathol 158(1):153-161

McGuire JK, Li Q, Parks WC (2003) Matrilysin (matrix metalloproteinase-7) mediates E-cadherin ectodomain shedding in injured lung epithelium. Am J Pathol 162(6):1831-1843

Mura M, Binnie M, Han B, Li C, Andrade CF, Shiozaki A, Zhang Y, Ferrara N, Hwang D, Waddell TK, Keshavjee S, Liu M (2010) Functions of type II pneumocyte-derived vascular endothelial growth factor in alveolar structure, acute inflammation, and vascular permeability. Am J Pathol 176(4):1725-1734

$\mathrm{Ng} \mathrm{HH}$, Narasaraju T, Phoon MC, Sim MK, Seet JE, Chow VT (2012) Doxycycline treatment attenuates acute lung injury in mice infected with virulent influenza H3N2 virus: Involvement of matrix metalloproteinases. Exp Mol Pathol 92(3):287-295

Nunes S (2005) Lung oedema in acute lung injury. Anaesthesia, pain, intensive care and emergency medicine., pp 345-355

Perrone LA, Plowden JK, Garcia-Sastre A, Katz JM, Tumpey TM (2008) H5N1 and 1918 pandemic influenza virus infection results in early and excessive infiltration of macrophages and neutrophils in the lungs of mice. PLOS Pathog 4(8):e1000115

Rhein LM, Perkins M, Gerard NP, Gerard C (2008) FcgammaRIII is protective against Pseudomonas aeruginosa pneumonia. Am J Respir Cell Mol Biol 38 (4):401-406

Rogers K (2010) The respiratory system. The rosen publishing group, New York USA

Steinberg BE, Goldenberg NM, Lee WL (2011) Do viral infections mimic bacterial sepsis? The role of microvascular permeability: A review of mechanisms and methods. Antiviral Res 93(1):2-15

van der Vries E, Schutten M, Boucher CA (2011) The potential for multidrug-resistant influenza. Curr Opin Infect Dis 24(6):599-604

van Riel D, Munster VJ, de Wit E, Rimmelzwaan GF, Fouchier RA, Osterhaus AD, Kuiken T (2007) Human and avian influenza viruses target different cells in the lower respiratory tract of humans and other mammals. Am J Pathol 171 (4):1215-1223

von Hundelshausen P, Koenen RR, Sack M, Mause SF, Adriaens W, Proudfoot AE, Hackeng TM, Weber C (2005) Heterophilic interactions of platelet factor 4 and RANTES promote monocyte arrest on endothelium. Blood 105(3):924-930

Wang S, Le TQ, Kurihara N, Chida J, Cisse Y, Yano M, Kido H (2010) Influenza virus-cytokine-protease cycle in the pathogenesis of vascular hyperpermeability in severe influenza. J Infect Dis 202(7):991-1001

Ware LB, Matthay MA (2000) The acute respiratory distress syndrome. N Engl J Med 342(18):1334-1349

Weber C, Fraemohs L, Dejana E (2007) The role of junctional adhesion molecules in vascular inflammation. Nature reviews 7(6):467-477

Yasui H, Kiyoshima J, Hori T (2004) Reduction of influenza virus titer and protection against influenza virus infection in infant mice fed Lactobacillus casei Shirota. Clin Diagn Lab Immunol 11(4):675-679

Zhang B, An J, Shimada T, Liu S, Maeyama K (2012a) Oral administration of Enterococcus faecalis FK-23 suppresses Th17 cell development and attenuates allergic airway responses in mice. Int J Mol Med 30(2):248-254

Zhang Y, Sun H, Fan L, Ma Y, Sun Y, Pu J, Yang J, Qiao J, Ma G, Liu J (2012b) Acute respiratory distress syndrome induced by a swine 2009 H1N1 variant in mice. PLoS One 7(1):e29347

Zhu L, Shimada T, Chen R, Lu M, Zhang Q, Lu W, Yin M, Enomoto T, Cheng L (2012) Effects of lysed Enterococcus faecalis FK-23 on experimental allergic rhinitis in a murine model. Journal of biomedical research 26(3):226-234

\section{doi:10.1186/2193-1801-2-269}

Cite this article as: Fukada et al: Enterococcus faecalis FK-23 affects alveolar-capillary permeability to attenuate leukocyte influx in lung after influenza virus infection. SpringerPlus 2013 2:269. 